\title{
ON SEMIPRIME RINGS OF BOUNDED INDEX
}

\author{
EFRAIM P. ARMENDARIZ
}

\begin{abstract}
A ring $R$ is of bounded index (of nilpotency) if there is an integer $n \geq 1$ such that $x^{n}=0$ whenever $x \in R$ is nilpotent. The least such positive integer is the index of $R$. We show that a semiprime ring $R$ has index $\leq n$ if and only if $R$ is a subdirect product of prime rings of index $\leq n$.
\end{abstract}

A ring $R$ is of bounded index if there is an integer $n \geq 1$ such that $x^{n}=0$ whenever $x$ is a nilpotent element of $R$. The least such positive integer is called the index of $R$, and we denote it by $i(R)$. V. A. Andrunakievic and Ju. M. Rjabuhin [1], and, independently, P. N. Stewart [6] have shown that a ring of index 1 (i.e. a ring with no nonzero nilpotent elements) is a subdirect product of integral domains. In this article we will show that a semiprime ring of index $n$ is a subdirect product of prime rings each of index at most $n$.

For a subset $X$ of a ring $R$ we let $r(X)$ denote the right annihilator of $X$, while $l(X)$ denotes the left annihilator of $X$. If $T$ is an ideal of a semiprime ring $R$, then it is easily seen that $l(T)=r(T)$; hence we write ann $(T)$ for this two-sided ideal of $R$.

We begin by adding one more equivalence to the following result of J. Hannah [4, Proposition 2].

LEMMA 1. Let $R$ be a semiprime ring, $n$ a positive integer. Then the following statements are equivalent.

(a) $i(R) \leq n$.

(b) If $X_{1}, \ldots, X_{n} \subseteq R$ such that $X_{i} X_{j}=0$ whenever $i \geq j$, then $X_{1} X_{2} \ldots X_{n}=$ 0 .

(c) If $X$ subseteqR, then $r\left(X^{n}\right)=r\left(X^{n+1}\right)$.

(d) For each $x \in R, r\left(x^{n}\right)=r\left(x^{n+1}\right)$.

LEMMA 2. Let $R$ be a semiprime ring and $n$ a positive integer. Then $i(R) \leq n$ if and only if

$$
\text { for each } x \in R \text { and each ideal } T \text { of } R, r\left(T x^{n}\right)=r\left(T x^{n+1}\right) \text {. }
$$

PROOF. If $(*)$ holds, then letting $T=R$ we get $r\left(R x^{n}\right)=r\left(R x^{n+1}\right)$. However $R$ is semiprime so $r(R a)=r(a)$ for all $a \in R$. Hence (d) of Lemma 1 is satisfied so that $i(R) \leq n$. Thus assume $i(R) \leq n$. We shall use Lemma $1(\mathrm{~b})$ to establish (*). Proceeding as in Hannah's proof, we let $X_{i}=r\left(T x^{i}\right) T x^{i}$ for $i=1,2, \ldots, n$. Then for $i \geq j$ we have $X_{i} X_{j}=0$ so we must have

$$
\begin{aligned}
0 & =X_{1} X_{2} \cdots X_{n}=T \cdot X_{1} X_{2} \cdots X_{n} \\
& =[T \cdot r(T x)]\left[T x \cdot r\left(T x^{2}\right)\right]\left[T x^{2} \cdot r\left(T x^{3}\right)\right] \cdots\left[T x^{n-1} \cdot r\left(T x^{n}\right)\right] T x^{n} .
\end{aligned}
$$

Received by the editors July 11, 1980.

1980 Mathematics Subject Classification. Primary 16A12, 16A48.

Key words and phrases. Bounded index, semiprime ring, prime rings, subdirect product.

(c) 1982 American Mathematical Society 0002-9939/81/0000-1056/\$01.75 
Now for each $i \geq 0$ (with $T x^{0}=T$ ) we have $T x^{n} \cdot r\left(T x^{n+1}\right)=T x^{i} \cdot x^{n-i} r\left(T x^{n+1}\right.$ ) and $x^{n-i} \cdot r\left(T x^{n+1}\right) \subseteq r\left(T x^{i+1}\right)$. Hence $T x^{n} \cdot r\left(T x^{n+1}\right) \subseteq T x^{i} \cdot r\left(T x^{i+1}\right)$ for $i \geq$ 0 . But then $\left[T x^{n} \cdot r\left(T x^{n+1}\right)\right]^{n+1}=0$ so $T x^{n} \cdot r\left(T x^{n+1}\right)=0$ since $R$ is semiprime. Thus $r\left(T x^{n+1}\right) \subseteq r\left(T x^{n}\right)$ and equality follows.

LEMMA 3. Assume $R$ is a semiprime ring of index $n$ and $T$ is an ideal of $R$. If $K=\operatorname{ann}(T)$ then $i(R / K) \leq n$.

Proof. Since $K=\operatorname{ann}(T), R / K$ is a semiprime ring. Let $\bar{R}=R / K$ and let $\bar{a}=a+K \in \bar{R}$. If $\bar{w} \in r\left(\bar{a}^{n+1}\right)$, then $a^{n+1} w \in K$ so $T a^{n+1} w=0$. Since $r\left(T a^{n+1}\right)=r\left(T a^{n}\right)$, we have $a^{n} w \in K$ and so $\bar{w} \in r\left(\bar{a}^{n}\right)$. By Lemma 1(c), $i(R / K) \leq n$.

THEOREM 1. Let $R$ be a semiprime ring of index $n$. Then $R$ is a subdirect product of prime rings of index $\leq n$.

PROOF. We must show that for each $a \in R, a \neq 0$, there is a prime ideal $P$ with $a \notin P$ and $i(R / P) \leq n$. Since $R$ is semiprime and $a \neq 0$, there is an $m$-system containing $a$ [5, Chapter 4]. Specifically let $a_{1}=a$; then $a_{1} R a_{1} \neq 0$ implies $a_{1} b_{1} a_{1}=a_{2} \neq 0$ for some $b_{1} \in R$. Inductively we obtain sequences $\left\{b_{i}\right\}$, $\left\{a_{i}\right\}$ with $a_{i+1}=a_{i} b_{i} a_{i} \neq 0$ and $\mathcal{M}=\left\{a_{i}\right\}$ is the desired $m$-system. Using Zorn's Lemma, the set $\{A \mid A$ an ideal of $R, \mathcal{M} \cap A=\emptyset$ and $i(R / A) \leq n\}$ has a maximal element $P$ which we claim is a prime ideal of $R$. To show that $P$ is a prime ideal, we first note that $P$ is a semiprime ideal of $R$. For if $P \subseteq W$ and $W^{2} \subseteq P$, then $i(R / W) \leq n$ since $i(R / P) \leq n$. Now if $P \subseteq W$, then $\mathcal{M} \cap W \neq \emptyset$ so $a_{i} \in W$ for some $i \geq 1$. But then $a_{i} R a_{i} \subseteq W^{2} \subseteq P$, so $a_{i+1} \in P$, a contradiction. Thus $P$ is indeed a semiprime ideal of $R$. By passing to $R / P$ we assume that $P=0$. If $R$ is semiprime but not prime, we have nonzero ideals $A, B$ with $A=\operatorname{ann}(B)$ and $B=\operatorname{ann}(A)$. Then by Lemma $3, i(R / A) \leq n$ and $i(R / B) \leq n$. Hence $\mathcal{M} \cap A \neq \emptyset$ and $\mathcal{M} \cap B \neq \emptyset$. But if $a_{i} \in \mathcal{M} \cap A, a_{j} \in \overline{\mathcal{M}} \cap B$ with $j \geq \bar{i}$, then $a_{j} \in A \cap B=0$, a contradiction. Thus $P$ is a prime ideal, as desired.

As a consequence we have the index 1 result.

THEOREM 2 (ANDRUNAKIEVIČ AND RJABUhIN; STEWART). A ring $R$ has no zero nilpotent elements if and only if $R$ is a subdirect product of integral domains.

In addition we deduce

THEOREM 3. Let $R$ be a ring of index $n$, and let $N$ denote the prime radical of $R$. Then $N=\bigcap\{P \mid P$ is a prime ideal, $i(R / P) \leq n\}$.

Proof. Since $N$ is a nil ideal, $i(R / N) \leq n$ and $R / N$ is semiprime so the statement follows.

Hannah also establishes in [4, Proposition 5] that a prime ring has index $\leq n$ if and only if each chain of left (right) annihilators has at most $n$ proper inclusions. Thus a prime ring of bounded index has ACC on left and right annihilators. As Hannah points out, however, semiprime rings of index $n$ need not have the ACC on left or right annihilators. (An infinite direct product of fields provides an appropriate example.) There is, however, a class of semiprime rings for which bounded index yields the ACC on left and right annihilators. A semiprime ring with unit is (right) strongly semiprime if each faithful ideal contains a finitely generated left ideal whose right annihilator is zero. A strongly prime ring is a prime ring 
which is strongly semiprime. For properties of these rings see [2, 3]. In particular D. Handelman shows [3, Theorem 1] that a ring $R$ is strongly semiprime if and only if $R$ is a finite subdirect product of strongly prime rings. Lemma 3 is instrumental in showing

THEOREM 4. A ring $R$ is strongly semiprime of index $\leq n$ if and only if $R$ is a finite subdirect product of strongly prime rings of index $\leq n$.

ProOF. According to [3, Theorem 1] the prime rings are obtained by taking $R / P$, where $P$ is maximal among the annihilators of two-sided ideals of $R$. If $R$ has index $n$, then, by Lemma $3, i(R / P) \leq n$. The converse is evident.

Using [4, Proposition 5] yields

THEOREM 5. If $R$ is a strongly semiprime ring of index $n$ then $R$ has $A C C$ on left and right annihilators.

\section{REFERENCES}

1. V. A. Andrunakievix and Ju. M. Rjabuhin, Rings without nilpotent elements and completely simple ideals, Dokl. Akad. Nauk SSSR 180, 9-11; English transl. in Soviet Math. Dokl. 9 (1968), 565-568.

2. J. Beachy and W. D. Blair, Rings whose faithful left ideals are cofaithful, Pacific J. Math. 58 (1975), 1-13.

3. D. Handelman, Strongly semiprime rings, Pacific J. Math. 60 (1975), 115-122.

4. J. Hannah, Semiprime rings with bounded index of nilpotence, Math. Res. Rep. No. 7, University of Melbourne, Melbourne, Australia, 1980.

5. N. H. McCoy, The theory of rings, Macmillan, New York, 1964.

6. P. N. Stewart, Semi-simple radical classes, Pacific J. Math. 32 (1970), 249-255.

Department of Mathematics, The University of TeXas, Austin, TeXas 78712 